\title{
Broadening the future of value account of the wrongness of killing
}

\author{
Ezio Di Nucci
}

\begin{abstract}
On Don Marquis's future of value account of the wrongness of killing, 'what makes it wrong to kill those individuals we all believe it is wrong to kill, is that killing them deprives them of their future of value'. Marquis has recently argued for a narrow interpretation of his future of value account of the wrongness of killing and against the broad interpretation that I had put forward in response to Carson Strong. In this article I argue that the narrow view is problematic because it violates some basic principles of equality and because it allows for some of the very killing that Marquis sets out to condemn; further, I argue that the chief reason why Marquis chooses the narrow view over the broad view - namely that the broad view would take the killing of some nonhuman animals to be also wrong - should rather be considered a welcome upshot of the broad view.
\end{abstract}

On Don Marquis's future of value account of the wrongness of killing, "what makes it wrong to kill those individuals we all believe it is wrong to kill, is that killing them deprives them of their future of value" (Marquis 1989, Marquis 2011). Recently Carson Strong $(2008,2009)$, Don Marquis (2011) and I (2009a, 2009b) have been arguing about a set of supposed counterexamples to the future of value account proposed by Strong, involving either a terminally ill patient or an individual severely and permanently cognitively impaired. Strong argues that it would be wrong to kill those individuals despite their not having a future of value like ours. I have argued that there are some serious interpretative problems with both Marquis's concepts of "future like ours" and "future of value" and with Strong's 
counterexample (Di Nucci 2009a and 2009b); and that, on a charitable interpretation that sets aside those problems, Strong's counterexamples fail because they involve ultimately unacceptable moral claims in violation of basic principles of equality: they involve, to put it simply, the claim that the individuals in the counterexamples do not have a valuable future.

I have distinguished (2009b) between a narrow interpretation of "future of value", according to which those individuals do not have a future of value; and a liberal interpretation of "future of value", which allows us to regard the future of terminally ill patients and severely cognitively impaired individuals as valuable. And I have argued that we must not interpret "future of value" as narrowly as Strong proposes - so that we can avoid his violation of basic principles of equality; and that a more liberal interpretation has the consequence that it will enlarge the domain of wrongful killing: we should welcome this outcome.

Marquis has responded by also distinguishing between a narrow and broad interpretation of his future of value account; arguing that, on the narrow view, Strong's terminally ill counterexample does not work - because the terminally ill patient does have a future of value qualitatively like ours, just shorter. But Marquis concedes that Strong's second counterexample, involving a severely and permanently cognitively impaired individual, does succeed - because on the narrow view the cognitively impaired individual does not have a future of value like ours. This counterexample would not work against a broader view of future of value, concludes Marquis. "According to the broad view, one has a future of value just in case, if not killed, one's future will consist, on balance, of experiences one will value" (Marquis 2011). 
While I argue (2009b) that we should adopt the more liberal interpretation of "future of value", Marquis opts for the narrow view, because "the narrow version does not even suggest that killing rabbits or mosquitoes may be wrong" (Marquis 2011). Here I argue, contra Marquis, that we should instead pursue the broader more liberal interpretation of "future of value". There are overwhelming reasons for it: first, the broad view does not violate basic principles of equality; second, the broad view is not subject to Strong's severely and permanently cognitively impaired counterexample; third, the broad view, in widening the domain of wrongful killing, is more inclusive and in this respect, I argue at the end of the paper, ethically preferable.

Let me emphasize the sorts of problems involved in endorsing the narrow view: the narrow view cannot account - by Marquis's own admission - for the wrongness of killing severely and permanently cognitively impaired individuals; and it therefore cannot account for the wrongness of killing foetuses that will develop into severely and permanently cognitively impaired individuals. Those foetuses, according to the narrow view, do not have a future of value. So they may be killed - or, at least, their killing is not wrongful. That is going to represent a substantial exception to the general rejection of abortion that Marquis (1989) put forward with his "future of value" account. It is wrong to kill some foetuses and it is not wrong to kill other foetuses. What's the difference? It is wrong to kill foetuses that have a "future of value"; while it is not wrong to kill foetuses that do not have a "future of value". This emphasizes in what sort of intractable moral territory the narrow view forces us: we are forced into the nasty business of evaluating futures (Di Nucci 2009b).

Now, it might be objected that this dirty work needs to be done. After all, recognising the differences between severely cognitively impaired individuals and healthy ones 
acknowledges the tragedy for those born with such impairments and their families. Those differences, the objection goes, must be emphasized. And that is painfully true. But the question is whether this difference should be emphasized in terms of moral value. That's where I think that the narrow view of "future of value" is in violation of basic principles of equality if it says or implies that some individuals are morally preferable or more valuable than other individuals. We may be as brutal as to say that the lives of severely cognitively impaired individuals are qualitatively inferior to ours. And I agree that such brutality is necessary, as a form of respect towards the lives of the severely cognitively impaired. But that is still not the same as saying that those lives are morally less valuable than our lives. It is not the same as saying that it is wrong to kill some foetuses and not wrong to kill others. And it is in this respect that I think the broad view should be preferred to the narrow view.

A note of clarification: it might be thought that I have overstated my argument by emphasizing that the narrow view would imply that it is wrong to kill some foetuses and not wrong to kill other foetuses. It might be proposed that, rather, all that the narrow view implies is that killing some foetuses is morally worse than killing other foetuses; that there are differences in the various moral evaluations of the different killings is not the same as saying that some killings are permissible while others are not. Those who might find it an unacceptable consequence of the narrow view that some foetuses may be killed while others may not, could on the other hand live with the weaker claim that the narrow view implies different moral evaluations for different killings. I agree that this latter proposal is importantly different and in this respect not as problematic as the former one, but I think that the narrow view implies the former claim, namely that killing some foetuses is wrong while killing others is not wrong. To see this, take Marquis's statement of the narrow view: 
Let us call our (ie, yours and mine, readers) futures of value 'pfutures of value'. P-futures are the kind of future lives that can be characterised as the lives of persons. I have a p-future. The fetus I once was had a p-future. (Note that this claim is a simple consequence of the way 'future of value' was defined and wellknown facts.) According to what I shall call 'the narrow view' valuable futures are futures like ours as long as they are $p$-futures of value... The severely retarded human beings to which Strong refers do not have $p$-futures of value. (2011)

Don has a p-future and it is therefore wrong to kill Don. The foetus that Don once was had a p-future and it was therefore wrong to kill that foetus. On the other hand John, a severely and permanently cognitively impaired 40 -year-old, does not have a $p$-future. The foetus that John once was did not have a p-future either. Therefore it is not wrong to kill John now, and forty years ago it would not have been wrong to kill the foetus that John then was. So the narrow view does imply that it is wrong to kill some individuals while it is not wrong to kill others. And it does imply that it is wrong to kill some foetuses while it is not wrong to kill others.

So the narrow view allows for too much killing, I have argued. But Marquis is rather worried that the broad view does not allow for enough killing - that's his reason for sticking to the narrow view. I think, contra Marquis, that we should welcome the moral restrictions of the broad view. Here I should first of all caution about the difficulty in evaluating the future of value account against the worry that it does not allow for enough killing. Recall the statement of the future of value account with which we started: "what makes it wrong to kill 
those individuals we all believe it is wrong to kill, is that killing them deprives them of their future of value" (Marquis 2011). The account is a bit more specific than just the wrongness of any killing. The future of value account is, specifically, about the wrongness of killing a certain class of individuals: those individuals we all believe it is wrong to kill.

In assessing the worry that the broad view does not allow for enough killing, we need to know who those individuals we all believe it is wrong to kill are. I guess Don Marquis is one of them. I am probably one too, as are most if not all the readers that Marquis addresses. Already when we move to the foetuses that the aforementioned individuals once were, it becomes trickier. But that's ok, because after all that was exactly the point of Marquis's original argument ${ }^{2}$ : not to have to deal directly with the status of the foetus, but rather with the much less controversial status of the future individuals in which foetuses normally develop. But what about John? Do we all believe it is wrong to kill John? Amongst philosophers it is perhaps unsurprising that we don't all agree (Singer 1975, 1979). But it seems that in society at large there is also no consensus. Let me be clear here that I am not saying that such consensus either within society at large or within philosophers would necessarily make any moral difference: I am only referring to it because such consensus is part of Marquis' argument for the wrongness of killing.

Why is this important? Individuating which individuals the future of value account of the wrongness of killing refers to is important because Marquis's reason to choose the narrow view against the broad view is that "the narrow version does not even suggest that killing rabbits or mosquitoes may be wrong" (2011). If society cannot agree on John, it certainly cannot agree on the killing of rabbits. Are rabbits individuals we all believe it is wrong to kill? No, they are not - it's a simple statistical fact about human opinion that we don't all believe 
it is wrong to kill rabbits, nor do we all believe it is not wrong to kill rabbits. In this respect, there is a simple solution for Marquis's worry with rabbits, and therefore a simple rebuttal of his argument in favour of the narrow view: if we should choose the narrow view over the broad view because the narrow view, as opposed to the broad view, does not suggest that killing rabbits may be wrong, then that is no reason to choose the narrow view over the broad view simply because the future of value account is not about rabbits; and therefore neither the narrow view of the future of value account nor the broad view of the future of value account suggest that it may be wrong to kill rabbits simply because the future of value account does not talk about rabbits.

In one sense, then, we are already finished: we have given overwhelming reasons against the narrow view; and we have refuted the stated reason against the broad view. But it might be objected that we should not be so strict in the application of the future of value account; after all, the objection might go, the account is only interesting in so far as it deals with more individuals than just those about whom we all agree - there might not be many of those around, I am afraid. Therefore we should be charitable about the future of value account, and at least also evaluate it as a general account of the wrongness of killing, and not only as a particular account of the wrongness of killing those individuals that we all believe it is wrong to kill.

Let us pay our dues to the principle of charity and evaluate the future of value account also as a general account of the wrongness of killing. What should we then say about the worry that the broad view of the future of value account does not allow for enough killing because it suggests that killing rabbits might be wrong? I think we should just embrace this worry, and consider this consequence of the future of value account as a positive upshot. Indeed, 
progress in animal ethics might suggest that a general account of the wrongness of killing might have to say something about non-human animals. A particular account of the wrongness of killing only those individuals that we all believe it is wrong to kill does well to avoid the intricate questions of animal ethics - as we said, it is a statistical fact about human opinion that there is no agreement on that point in either philosophical circles or society at large. But a general account of the wrongness of killing has to say something about nonhuman animals, especially now that animal ethics has earned its place within moral philosophy. So it is a further advantage of the broad view that it accounts for the wrongness of killing non-human animals.

Someone might be worried that we have gone too far: they might accept that including some non-human animals amongst those to which the account applies might be a welcome consequence, but object that the problem for the future of value account arises when it can no longer distinguish between the wrongness of killing an healthy adult human being and the wrongness of killing a rabbit. So that the application of the future of value account to non-human animals might be seen as a reductio of the original argument. This reductio could take two forms:

1) future of value reasoning shows that it is wrong to kill non-human animals. But it is absurd to think that it is wrong to kill non-human animals. So future of value reasoning must be flawed.

2) Future of value reasoning shows that it is just as wrong to kill a non-human animal as it is to kill a human. It is absurd to think that it is just as wrong to kill a non-human animal as it is to kill a human. So future of value reasoning must be flawed. 
We have already addressed (1). Two points here about (2): first, many might actually want to go that far and welcome an account that does not distinguish between the wrongness of killing humans and the wrongness of killing non-human animals. Looking at the animal ethics literature suggests that an account such as the one (2) criticises would not be taken to be going particularly far (Singer 1975, Singer 1979, Regan 1984).

Secondly, I don't think that the broad view of the future of value account must imply that there is no moral difference between killing humans and killing animals. Recall the statement of the broad view: "According to the broad view, one has a future of value just in case, if not killed, one's future will consist, on balance, of experiences one will value" (Marquis 2011). That seems to embrace at least some non-human animals (Allen 2004). But it does explicitly refer to the subjective evaluation of one's experience. The point about the broad view is, indeed, that we are no longer evaluating and comparing futures and experiences across different individuals; it is enough that the one individual values (or would value) her future experiences - and therefore including some animals among those that it is wrong to kill does not violate basic principles of equality. ${ }^{1}$

This still leaves room for various possibilities, all of them short of the view that there is no moral difference between killing humans and killing non-human animals. It may be that the sense in which humans value their experiences is not available to non-human animals. That is, for example, where many introduce consciousness: it is not as if non-human animals do not have phenomenal experiences (think of pain); it is rather that their experiences are not conscious or self-conscious the way human experience is (Carruthers 1989). One might think

\footnotetext{
${ }^{1}$ Thanks to an anonymous referee for pressing me on this point.
} 
that this is a relevant moral difference: not enough to deny that it is wrong to kill some nonhuman animals (because they have the experience of pain, for example); but enough to deny that there is no significant moral difference between killing humans and killing animals. Alternatively, one classic strategy (from Mill's (1863) higher-lower pleasure distinction) is to distinguish between the quality of human experience and the quality of animal experience: again, that might suggest that there is a moral difference between killing humans and killing animals. Still, killing animals would not be morally irrelevant. So there is no reason to think that the broad view of the future of value account will lead to conclusions about killing animals that some might hold to be implausibly demanding. This also guarantees some common ground such that we do not need to accept that making some distinctions will violate basic principles of equality.

Summing up, I have argued against Marquis's argument for the narrow interpretation over the broad interpretation of the 'future of value' account of the wrongness of killing; and I have argued for an earlier suggestion ${ }^{6}$ in the literature according to which we should embrace the more liberal version of the future of value account. I have motivated this move by raising some serious problems with the narrow view and by arguing that the implications of the broad view, in particular with regards to the killing of non-human animals, are not problematic and should, rather, be welcomed. ${ }^{2}$

\footnotetext{
${ }^{2}$ I realize that I have here dealt very quickly with some highly controversial topics in bioethics, such as for example abortion. Please see my other bioethical writings for more in depth analysis of some of these issues (Di Nucci 2011, Di Nucci 2013a, Di Nucci 2013b, Di Nucci 2013c, Di Nucci 2013d, Di Nucci 2013e, Di Nucci 2014a, Di Nucci 2014b, Di Nucci 2014c, Di Nucci 2014d).
} 


\section{References}

Allen C. 2004. Animal Pain. Nous 38:617-43.

Carruthers P. 1989. Brute Experience. J Philos 86:258-269.

Di Nucci E. 2009a. Abortion: Strong's counterexamples fail. J Med Ethics 35:306-7.

Di Nucci E. 2009b. On how to interpret the role of the future within the abortion debate. J Med Ethics 35:651-52.

Di Nucci E. 2011. Sexual Rights and Disability. Journal of Medical Ethics 37 (3): 158-161.

Di Nucci E. 2013a. Killing Fetuses and Killing Newborns. Journal of Medical Ethics 39 (5): e19e20.

Di Nucci E. 2013b. Habits, Nudges, and Consent. American Journal of Bioethics 13 (6): 27-29.

Di Nucci E. 2013c. Embryo Loss and Double Effect. Journal of Medical Ethics 39 (8): 537-540.

Di Nucci E. 2013d. Withdrawing artificial nutrition and patients' interests. Journal of Medical Ethics 39 (9): 555-556.

Di Nucci E. 2013e. Self-Sacrifice and the Trolley Problem. Philosophical Psychology 26 (5): $662-672$.

Di Nucci E. 2014a. Addiction, Compulsion, and Agency. Neuroethics 7 (1): 105-107.

Di Nucci E. 2014b. Contraception and Double Effect. American Journal of Bioethics 14 (7): 4243.

Di Nucci E. 2014c. Fathers and Abortion. The Journal of Medicine \& Philosophy 39 (4): 444458.

Di Nucci E. 2014d. Ethics Without Intention. London: Bloomsbury. Marquis D. 1989. Why abortion is immoral. J Philos 86:183-202. 
Marquis D. 2011. Strong's objections to the future of value account. J Med Ethics 37:384388.

Mill J.S. 1863. Utilitarianism.

Regan T. 1984. The Case for Animal Rights. Berkeley: University of California Press.

Singer P. 1975. Animal Liberation. New York: New York Review Book.

Singer P. 1979. Practical Ethics. Cambridge: Cambridge University Press.

Strong C. 2008. A critique of 'The best secular argument against abortion'. J Med Ethics $34: 727-31$.

Strong C. 2009. Reply to Di Nucci: why the counterexamples succeed. J Med Ethics 35:326-7. 